\title{
The rise of regional programmes on critical infrastructure resilience: identification and assessment of current good practices
}

\author{
P. Trucco ${ }^{1}$, B. Petrenj ${ }^{1}$, S. Bouchon ${ }^{2}$ \& C. Di Mauro ${ }^{2}$ \\ ${ }^{1}$ Politecnico di Milano - School of Management, Italy \\ ${ }^{2} R G S$, Risk Governance Solutions S.r.l., Italy
}

\begin{abstract}
Effective Critical Infrastructure Protection and Resilience (CIP-R) depends on numerous stakeholders collaborating at different institutional and operational levels and exchanging information by means of a variety of channels. In this regard regional programmes or partnerships, have emerged as one of the key strategies to deal with CIP-R and Emergency Management (EM) issues effectively. Previous research has set the theoretical base of Public-Private Partnerships (PPP) and claimed their high potential for enhancing CIP-R that is vastly unexploited due to challenges in their establishment and management. It is now necessary to move forward to studying practical side of these programmes. MIRACLE (Multi-level Alignment of Regional Approaches to CI Resilience by Learning from Experience) is a research project funded by EC - DG HOME that aims at supporting regional CIP-R strategies in order to improve exiting capacities of the EU Member States to prevent, prepare and protect people against security related risks. This study presents a review of existing Good Practices (GPs), i.e. tools, technologies, activities and processes that are able to support: i) Establishment and management of regional PPPs for CIP-R; ii) Achievement of their main objectives and specific goals. The collected GPs are used to improve resilience levels in different phases of EM. GPs have been identified through an online survey, institutional websites, insights from professionals, available reports, documents and scientific literature. Finally, through engagement of international experts, professionals and researchers the GPs have been evaluated along three main parameters: implementation effort, transferability potential, type and relevance of expected benefits.
\end{abstract}

Keywords: critical infrastructure, resilience, good practices, regional, PPP. 


\section{Introduction}

To protect national infrastructure many public safety and other governmental agencies are establishing partnerships with private-sector organizations to assist in planning, resource allocation, communication strategy, and coordinated response to and strategic recovery following all types of hazards [1]. Regional programmes to Critical Infrastructure Protection and Resilience (CIP-R) aim to increase capacity to prevent, protect against, respond to, and recover from major incidents.

PPP models range from big cities, counties/provinces, regions, states/nations all the way to international. In the present study 'regional' is understood as the administrative scale but also as the coherent territory corresponding to a CI system extension.

The aim of the paper is to study existing regional CIP-R programmes and provide a state-of-the-practice analysis when it comes to successful practices in place. It presents a collection of good practices able to support implementation and functioning of regional CIP-R programmes. The practices were also evaluated by experts in the field, to assess their transferability, required effort and expected benefits. The main assumption behind the study is that, if regional CIP-R strategies are properly set and supported, they will allow addressing CIP$\mathrm{R}$ issues at higher levels (e.g. national or continental). Thus, the present study wants to contribute to a better understanding of this emerging phenomenon towards its full alignment with existing CIP policies and strategies.

\section{Definition of good practices and related research in the crisis management field}

Good practices are generally defined as 'Commercial or professional procedures that are accepted or prescribed as being correct or most effective' [2]. It is any collection of specific methods that when applied solve an existing problem, produce expected results and bring benefits. In our study it applies to available knowledge to addressing:

- Establishment and management of regional PPPs for Critical Infrastructure Protection and Resilience;

- The achievement of their main objectives and specific goals.

The rationale is to use a practice that have been effective in addressing similar issues in the past and apply it to a current problem. Still, more than often good practices (or 'best practices') offer insight into solutions that may or may not work for a given situation [3]. It can easily happen that a best practice is not applicable, is inappropriate for a particular case or just does not work well as in the original use. Ambler [4] offers an alternative view, 'contextual practice', in which the notion of what is 'best' will vary with the context. Not only the best practice often has to be adjusted for the new application but it can also evolve into its better version as improvements are discovered. Our work is a 'Good Practice' (GP) collection focus is on successful Activities, Procedures, Tools, and Technologies. 
The study of principles and good practices for public-private collaborations in the crisis management and resilience areas is not new. PADRES (Publicly Accessible, Dedicated, Resourced, Engaged, Sustainable) model lays out the set of essential attributes to assess/measure 'state-of-practice' of PPPs in EM [5]. The PADRES model has subsequently been used then to evaluate maturity levels and capabilities of different PPP levels/sizes across the US.

ENISA's (The European Network and Information Security Agency) efforts in this field focus on trying to analyse, understand and promote the models of PPPs at national and pan European levels. In 2009, ENISA issued its Good Practice Guide (GPG) on Information Sharing aimed at assisting Member States and other relevant stakeholders in setting up and running Network Security Information Exchanges in their own countries. In 2011, ENISA's Good Practice Guide [6] classified PPPs for security and resilience and revealed the main five components addressing Why, Who, How, What and When questions associated with creating and maintaining PPPs. ENISA offers high-level recommendations to stakeholder on how to successfully build PPPs (for resilient IT security in their context).

BUCOPCI project's Business Continuity Best Practices Report [7] and Security Plan Best Practices Report [8] were based on responses of six CI Operators on Spanish territory. In those reports the project investigated the percentage of compliance to the $B C M$ principles/standards [7] and level of compliance to the Operational Security Management among CI Operators [8].

RECIPE (Recommended Elements of Critical Infrastructure Protection for Policy Makers in Europe) Project's Good Practice Manual on CIP Policies [9] brings a set of GPs for covering areas of interest in CIP policies. Those include Identification of CI, Dependencies, PPPs, Information Sharing, Risk Management and CIP, Crisis Management and CI. Three dimensions that have a strong influence on the attainability of a large part of the practices have been considered:

- Involvement of private parties;

- Mandated or voluntary co-operation structure;

- $\quad$ Required CIP maturity.

\section{Study methodology}

The primary source of data was the MIRACLE project online survey aimed to review the existing Public-Private collaboration schemes (applied to address CIP-R issues) and to characterise the main features and modes of collaboration for further assessment and gap analysis. The scope of the survey was much broader than the aim of identifying potential GPs. Leveraging on the 159 survey responses, we were able to identify active regional CIP-R programmes and collect additional information such as references - public documents, reports and web-presentations, and direct contacts of the people involved in existing regional programmes (Figure 1).

The secondary source of data was digging into scientific and professional literature and Institutional websites. 


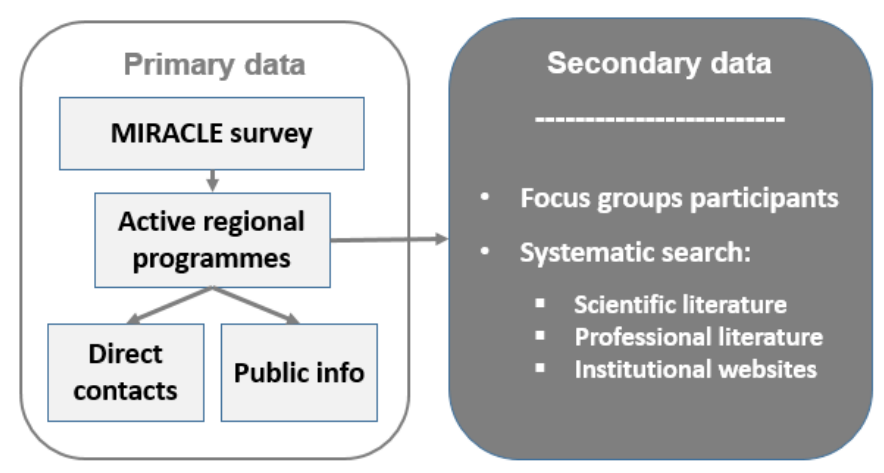

Figure 1: Data collection process.

More detailed information about identified GPs (both from the survey and from the secondary sources) were then collected through available public information or direct contact with people involved in these practices, mainly through focus groups and/or interviews. The final collection contains $21 \mathrm{Good}$ Practices for which a summary, highlighting main characteristics, is given in Tables 2-3.

\subsection{Identification and selection of GPs from the survey}

When identifying GPs (and active regional programmes) from the survey answers, we adopted criteria according to the study scope. In the first place, we filtered organisations involved in CIP-R collaborative activities or a programme, resulting in 92 organisations out of the 122 mapped in the survey. The focus was on programmes with the objective of improving resilience and/or Emergency Management (EM) in general, in contrast to ones with other main goals (e.g. enhancing service quality); in this way, 76 organisations in 17 different regions were selected. Focusing on these collaboration clusters we further searched for the available information on partnerships and practices in use. In order for a practice to qualify as GP, an organisation must have reported benefits from its adoption in terms of improvement of CIP-R related performance. Organizations that achieved to establish information sharing and stakeholder collaboration process have a significant importance in contrast to the ones that had no success. Finally, we tried to ensure (as much as possible) the coverage of GPs applied in different contexts (i.e. Regional, County/Province, Metropolitan Area, and City) and across all the EM phases (Mitigation, Preparedness, Response and Recovery).

\subsection{Secondary sources}

Search for GPs was also conducted inside Critical Infrastructure Warning Information Network (CIWIN), which is an initiative of the Directorate-General for Home Affairs of the European Commission. The search included repositories 
of National researches, FP6, FP7, CIPS and ISEC projects, their reports, deliverables and websites. Most of the developed tools focus on the technical aspects of communication/info-sharing and have not been embedded in a practical use (mostly in pilot tests of their functionality). These tools fall out of our scope since we are looking for practices that have been successfully used in practice and potentially transferable to another place. Another line of search for GPs included scientific and professional literature where we were able to find some related contributions. An overview of selected GPs is given in Section 4.

\subsection{Validation by international experts}

The GPs were assessed through engagement of a panel of international experts, professionals and researchers willing to perform as evaluators. They were provided with the full report on GPs and additional information was made available online. The online survey required approximately $15 \mathrm{~min}$, after previous reading of the GPs collection. It assessed the GPs across three dimensions associated with a specific Likert scale, ranging from 1 to 5:

- Benefits brought by these Good Practices to the implementing organizations and to the PPP as a whole;

- Effort and knowledge required to implement the following Good Practices in an organization or PPP;

- Level of Transferability of these Good Practices across different organizations, PPPs and regions.

The responses were collected over one month, during November and December 2014, and the survey received 20 responses, 13 of which complete.

\section{Classification and description of selected Good Practices}

In context of CIs, resilience in general implies 'the ability to reduce the magnitude and/or duration of disruptive events. The effectiveness of a resilient infrastructure or enterprise depends upon its ability to anticipate, absorb, adapt to, and/or rapidly recover from a potentially disruptive event' [10]. Comprehensive resilience considers all hazards, all EM phases, all stakeholders and all impacts relevant to disasters [1]. It requires capabilities to dynamically prevent, protect against, mitigate, respond to, and recover from the threats and hazards that pose the greatest risk. Enhancing resilience through improving Emergency Management requires a partnership among different levels of government and the private sector, detailed planning and co-operation among infrastructure sectors. EM is the discipline of avoiding and dealing with disasters in order to lessen their impact. [1]. A commonly used model of Comprehensive EM, firstly introduced by State Governors' Association in the US [11], encompasses four elements - mitigation (prevention), preparedness, response (coping) and recovery (aftermath). We have mapped GP contribution to resilience according to EM phases (Table 1). 


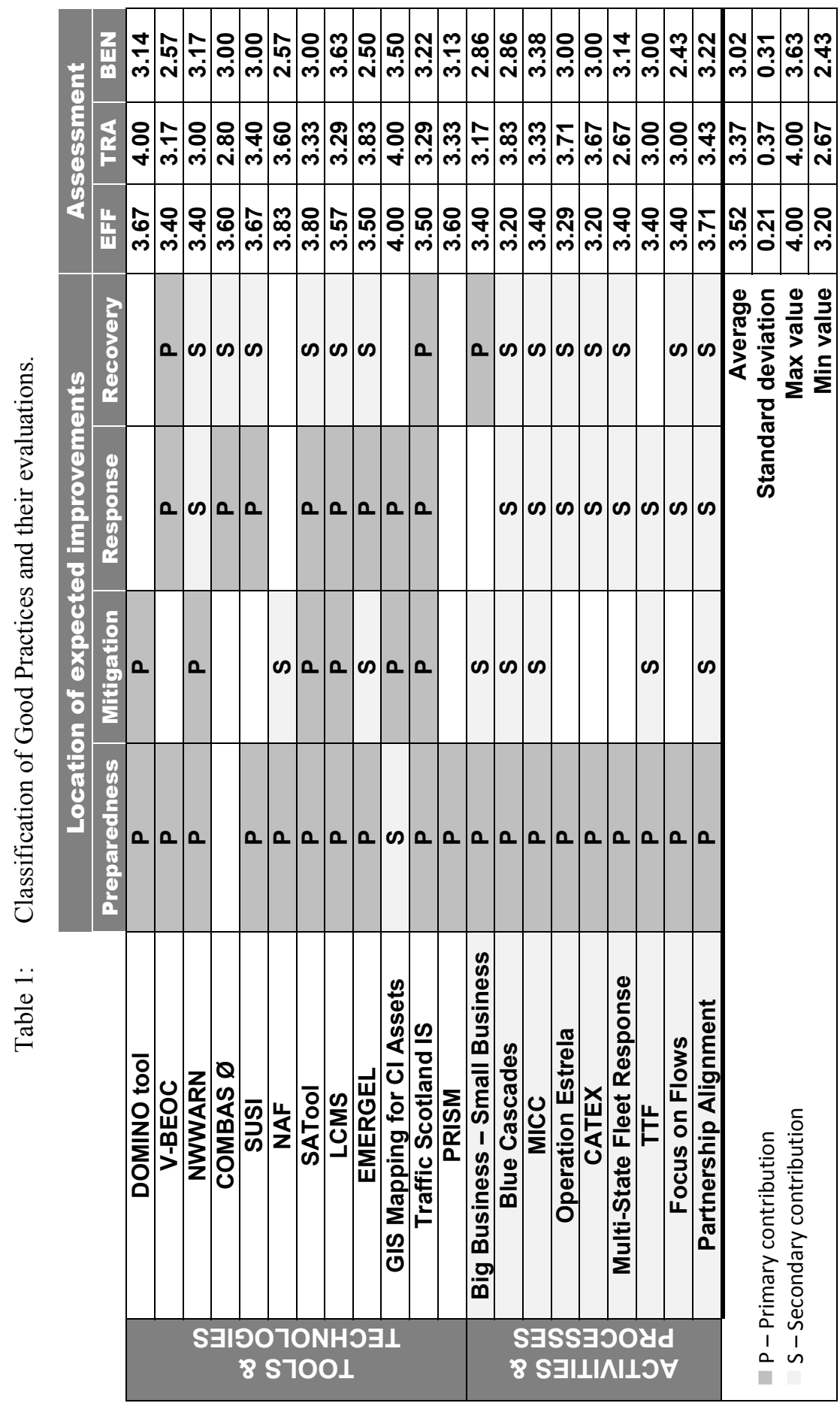




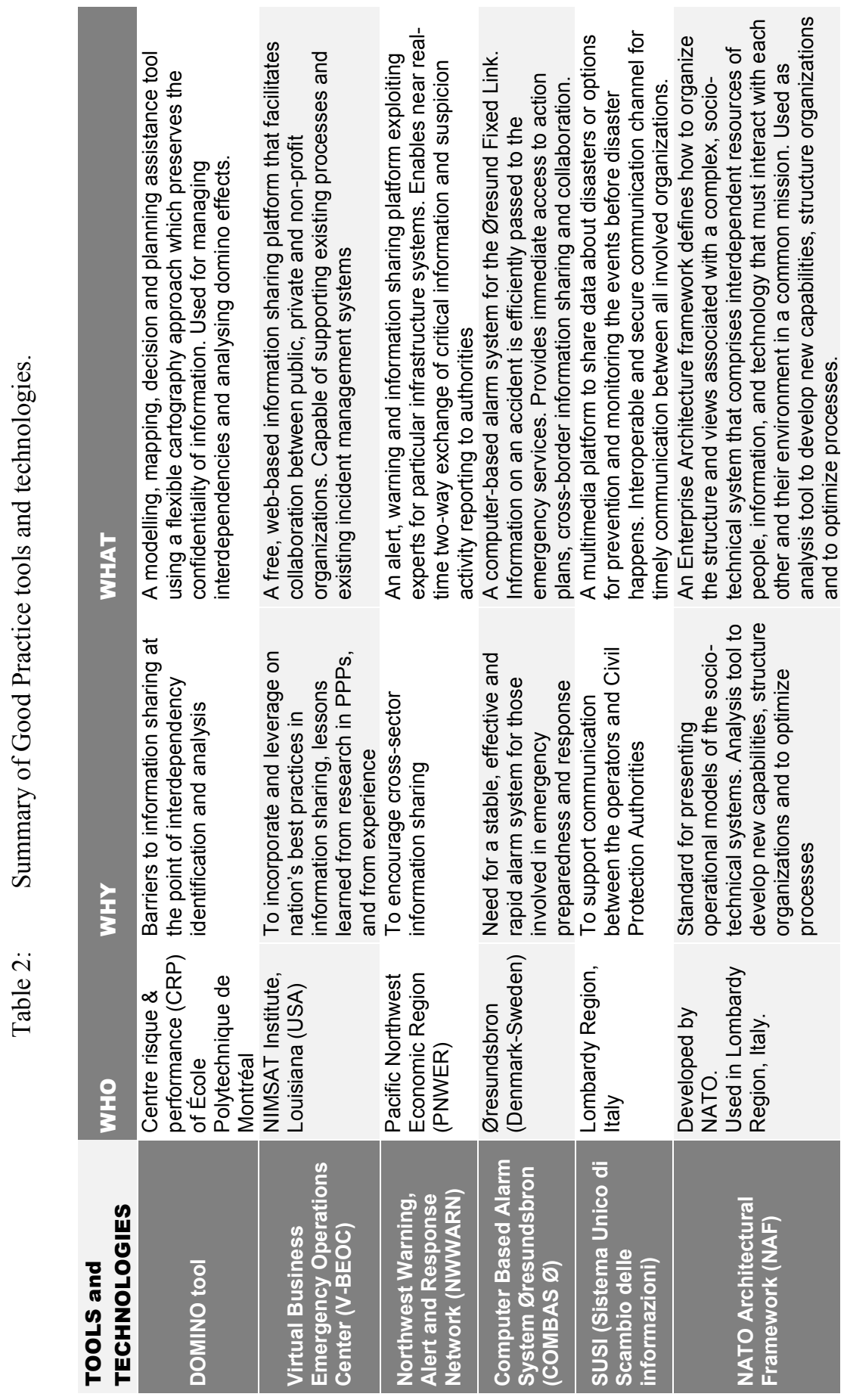




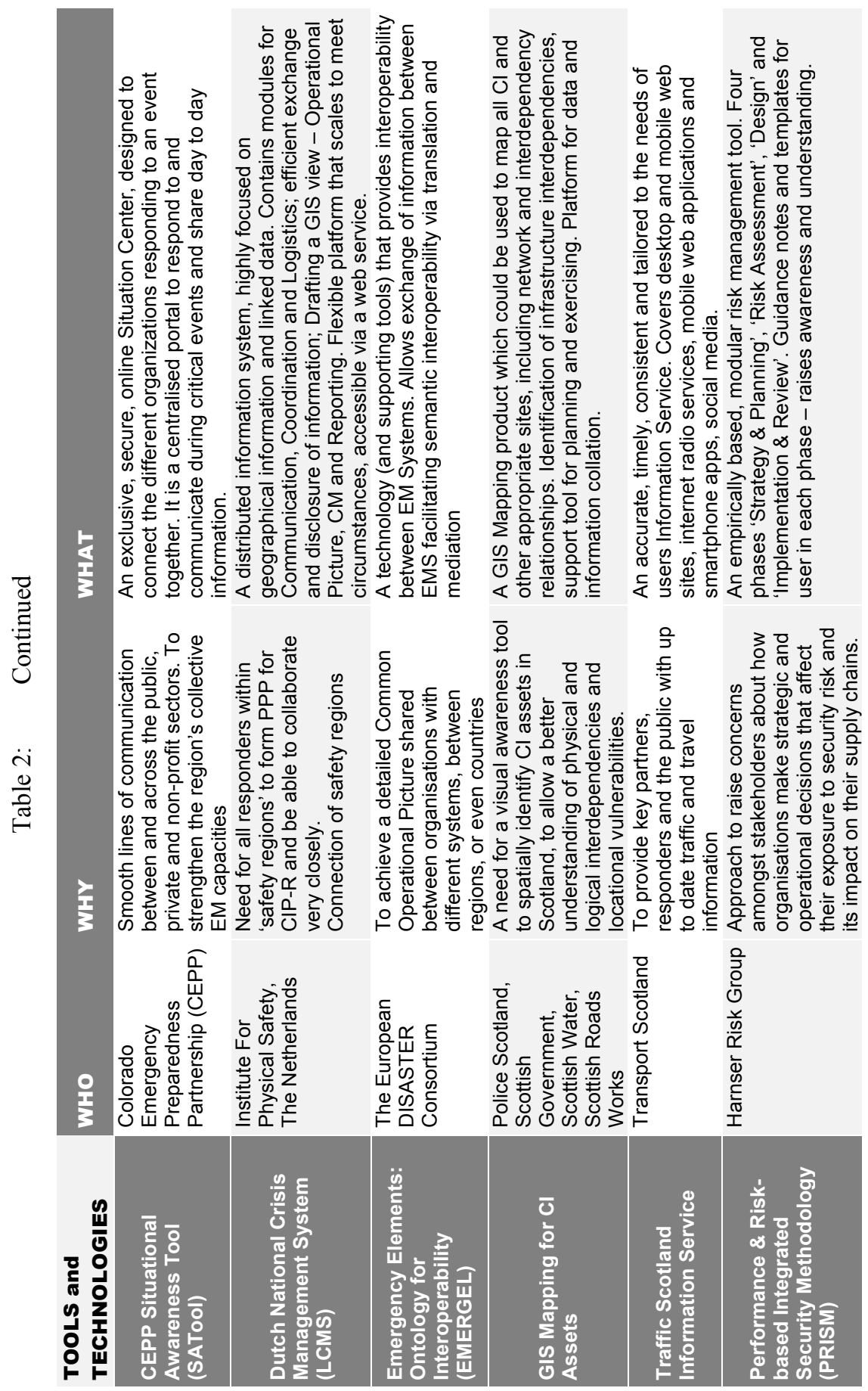

WIT Transactions on The Built Environment, Vol 150, (C) 2015 WIT Press www.witpress.com, ISSN 1743-3509 (on-line) 


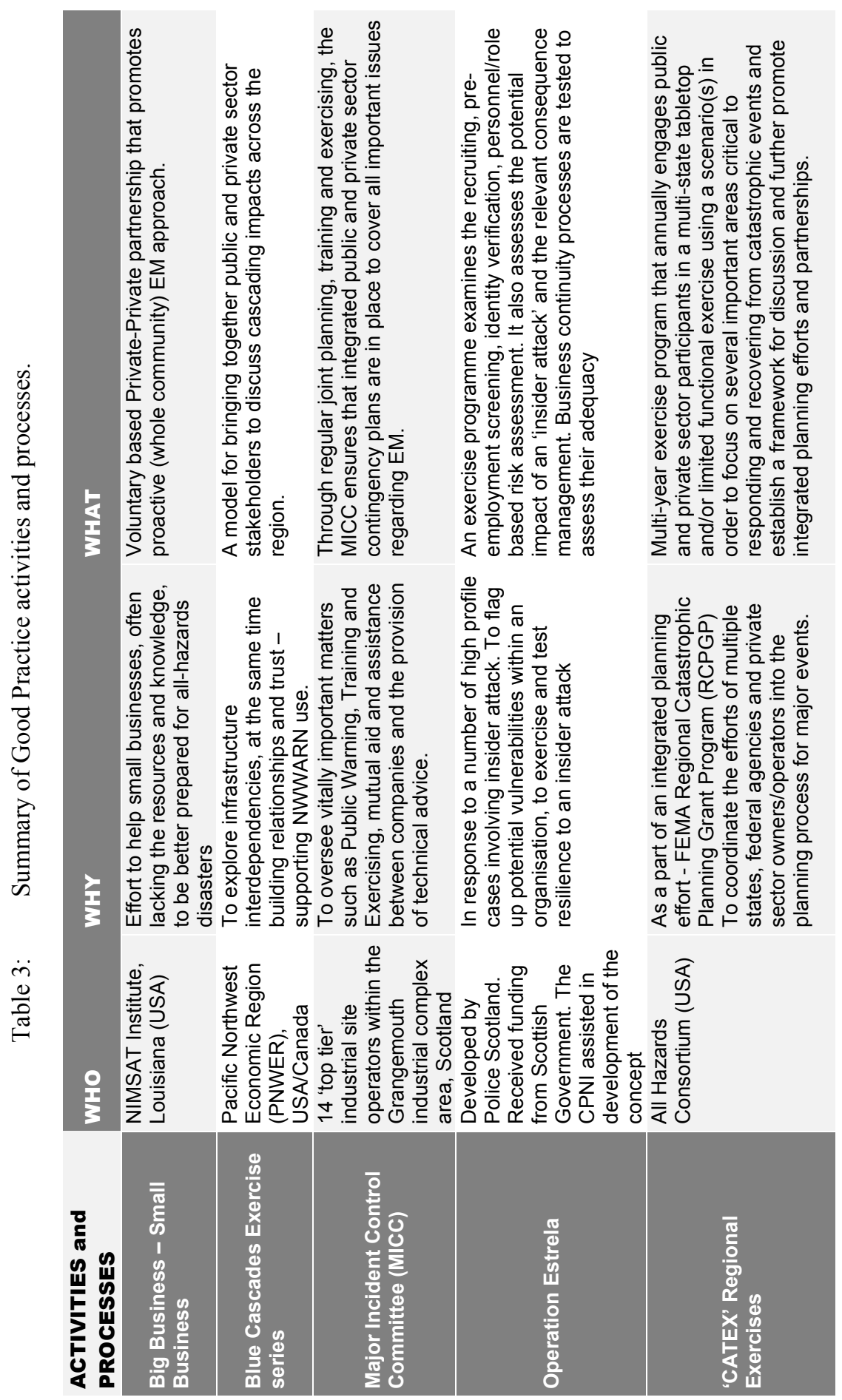




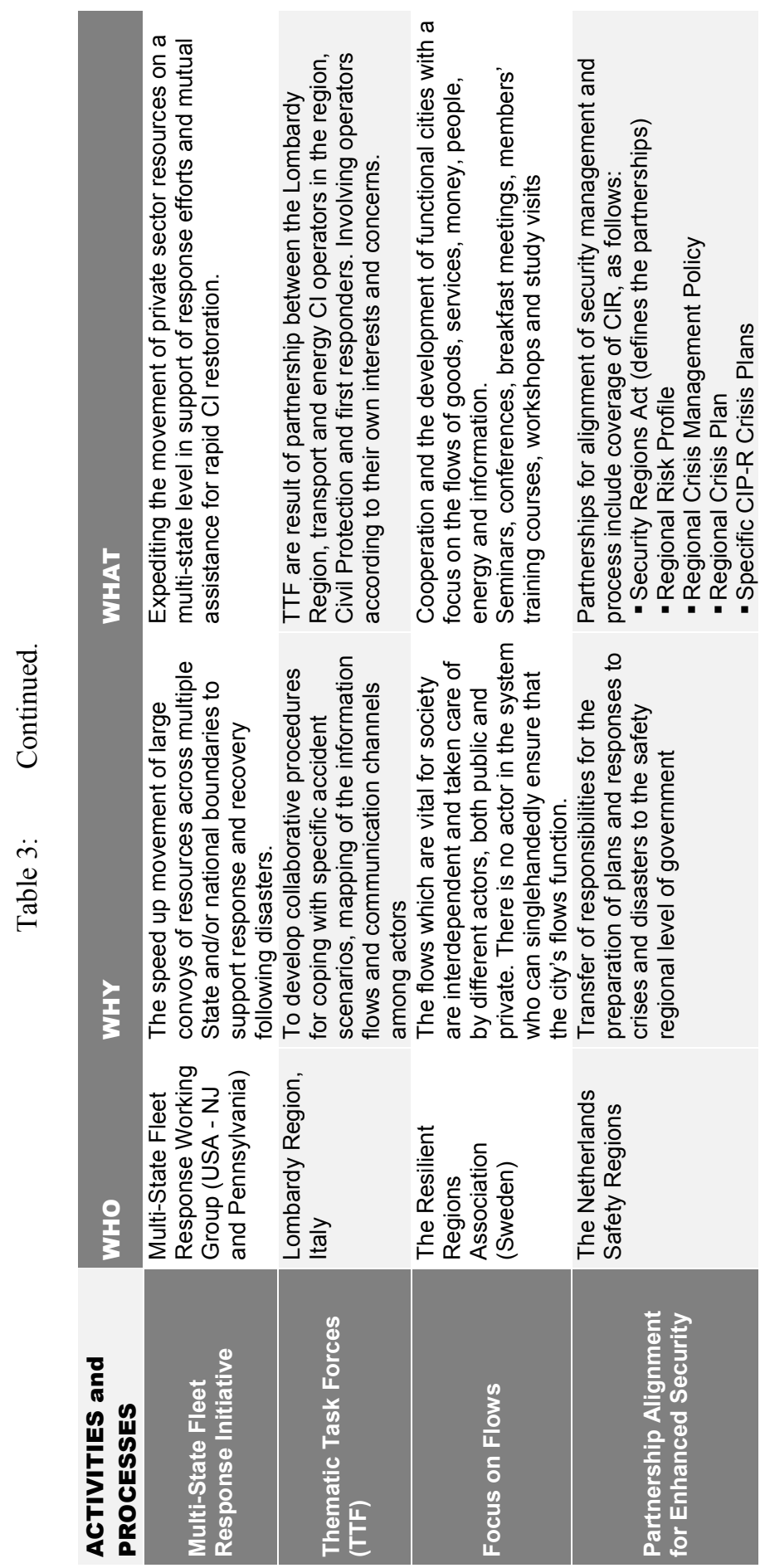

WIT Transactions on The Built Environment, Vol 150, (C) 2015 WIT Press www.witpress.com, ISSN 1743-3509 (on-line) 


\section{Assessment of Good Practices}

The average values for each GP across the three dimensions are shown in Table 1. All the practices received medium-high values of Benefits and Transferability, which approves them as Good Practices. The results of the GPs assessment exercise are plotted in Figure 2, where the size (width) of the bubble represents the benefits. As the GPs assessments fall quite close to each other, the average values have been rescaled (normalized) for a better representation and distinction.

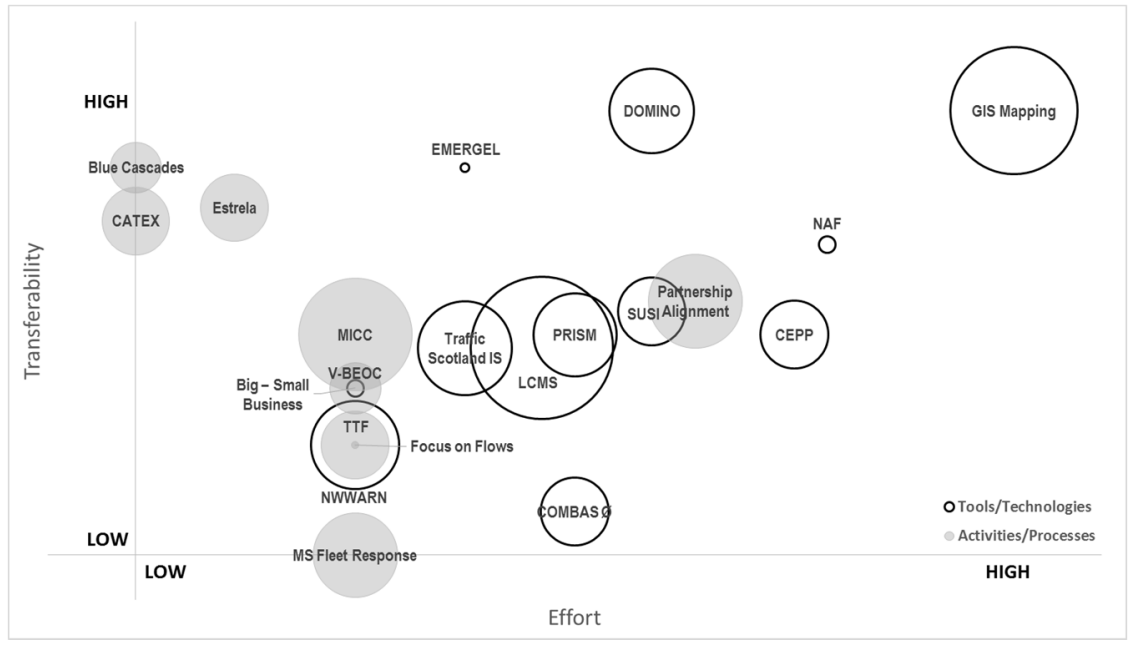

Figure 2: $\quad$ GPs assessment.

The experts feel that fairly enough effort and knowledge is required when it comes to implementing the GPs, which indicates a lack of 'quick wins'. It is understandable that Activities and Processes require less implementation effort compared to Tools and Technologies, while the latter are able to bring greater benefits and, on average, possess higher transferability. Even though some of the practices share the same guiding principles, each is significantly customised, addressing the specific needs of the regional programme. As practices are rarely directly transferable ('as such'), offering a hands-on experience would give practitioners a much better insight - an opportunity to examine the suitability for use, potential benefits, need for adjustments and human training in their own context. Feasibility of providing a first-hand experience is an issue to consider.

While GPs are usable when there is a lack of a practice, looking at the ratio between the benefits and effort it is clear that it would be irrational for any region to substitute a practice already in place with another one of the same type. Of course it does not mean that sharing of GPs between existing regional programmes is not needed. A reasonable action in this case would be to improve 
or expand the current practice based on the shared knowledge and experience and advance the existing CIP-R programme. GPs are not only a customised approaches but the ideas that lie behind the practices - the reasoning on how to cope with problems at hand and the base made of common determinants and features. GPs are therefore a source of knowledge on how to address issues, how to improve practices already in place and adopt new ones.

\section{Conclusions}

The paper studied the regional CIP-R programmes and provided a state-of-thepractice analysis when it comes to successful practices used for establishing and running a regional programme and reaching specific goals. After presenting a collection of GPs, we have involved experienced practitioners to evaluate those GPs along three relevant characteristics. The practitioners can dig into this GPs collection in search for ideas and solutions for their existing problems, leveraging on others experience and experts' opinions. Results may also support policy makers and practitioners to frame and develop regional CIP-R Programmes.

Future research will test implementation of some of the GPs into new environments. Determinants of GP, which are the primary transferable elements (rather than practices as such) should be extracted. Possible alignments and synergies should be further investigated in two aspects: i) Between the two types of GPs (Activities/Processes and Tools/Technologies) - for better mix and match; ii) Between programmes on different levels - i.e. in which way are regional CIP-R strategies able to address CIP-R issues at higher levels, and vice versa, how can CIP-R policies and strategies support a bottom-up approach in the form of regional programmes.

\section{Acknowledgement}

The present study has been completed under MIRACLE, a research project cofunded by DG Home Affairs of the European Commission, under CIPS/ISEC Work Programme. The financial support is gratefully acknowledged.

\section{References}

[1] FEMA website: https://training.fema.gov/EMI/

[2] Dictionary, O. E. Oxford dictionary of English. Online version, 2012.

[3] Bardach, E. A Practical Guide for Policy Analysis: The Eightfold Path to More Effective Problem Solving. Thousand Oaks, CA: Sage, 2011.

[4] Ambler, S. "Questioning 'Best Practices' for Software Development". Retrieved 17 November 2011.

[5] Federal Emergency Management Agency (FEMA). After Action Report of the first national conference on "Building Resilience through PublicPrivate Partnerships", August 3-4, 2011, Washington, D.C. 
[6] The European Network and Information Security Agency (ENISA). Cooperative Models for Effective Public Private Partnership - Good Practice Guide, 2011.

[7] Business Continuity Planning for Critical Infrastructures (BUCOPCI) Project. D1.2: Business Continuity Best Practices Report, 2012.

[8] Business Continuity Planning for Critical Infrastructures (BUCOPCI) Project. D1.3: Security Plan Best Practices Report, 2012.

[9] Recommended Elements of CIP for Policy Makers in Europe (RECIPE). Good Practices Manual for CIP Polices, 2014.

[10] National Infrastructure Advisory Council (NIAC) 'Critical Infrastructure Resilience - Final Report and Recommendations', U.S. Department of Homeland Security, Washington, D.C., 2009.

[11] National Governors' Association. Emergency preparedness project: Final report. Washington, DC: National Governor's Association, 1978. 